\title{
Mutagenesis of the FAE1 genes significantly changes fatty acid composition in seeds of Camelina sativa
}

\author{
Authors: Mahmet E. Ozseyhan, Jinling Kang, \\ Xiaopeng Mu, and Chaofu Lu
}

NOTICE: this is the author's version of a work that was accepted for publication in Plant Physiology and Biochemistry. Changes resulting from the publishing process, such as peer review, editing, corrections, structural formatting, and other quality control mechanisms may not be reflected in this document. Changes may have been made to this work since it was submitted for publication. A definitive version was subsequently published in Plant Physiology and Biochemistry, v. 123, (February 2018), DOI\# 10.1016/i.plaphy.2017.11.021

Ozseyhan, Mehmet E., Jinling Kang, Xiaopeng Mu, and Chaofu Lu. "Mutagenesis of the FAE1 genes significantly changes fatty acid composition in seeds of Camelina sativa." Plant Physiology and Biochemistry 123 (February 2018): 1-7. DOI: 10.1016/j.plaphy.2017.11.021.

Made available through Montana State University's $\underline{\text { ScholarWorks }}$ scholarworks.montana.edu 


\title{
Mutagenesis of the FAE1 genes significantly changes fatty acid composition in seeds of Camelina sativa
}

\author{
Mehmet E. Ozseyhan ${ }^{\mathrm{a}}$, Jinling Kang ${ }^{\mathrm{a}}$, Xiaopeng $\mathrm{Mu}^{\mathrm{a}, \mathrm{b}}$, Chaofu $\mathrm{Lu}^{\mathrm{a} \text {,* }}$ \\ ${ }^{a}$ Department of Plant Sciences and Plant Pathology, Montana State University, Bozeman, MT 59717, USA \\ ${ }^{b}$ College of Horticulture, Shanxi Agricultural University, Taigu, Shanxi 030801, China
}

\begin{abstract}
A B S T R A C T
Camelina sativa is a re-emerging low-input oilseed crop that has great potentials. It is necessary to ameliorate camelina oils for optimized fatty acid composition that can meet different application requirements. Camelina seed contains significant amounts of C20-C24 very long-chain fatty acids (VLCFAs) that may not be desirable. We demonstrated that these VLCFAs can be effectively reduced by deactivating the Fatty Acid Elongase1 (FAE1) in camelina. The allohexaploid camelina contains three alleles of FAE1 genes. Ethyl methanesulfonate (EMS) induced mutation at the FAE1-B gene caused over $60 \%$ reduction of VLCFAs in seed. Homozygous knockout mutants were successfully created in a single generation by simultaneously targeting three FAE1 alleles using the CRISPR technology with an egg cell-specific Cas 9 expression. VLCFAs were reduced to less than $2 \%$ of total fatty acids compared to over $22 \%$ in the wild type, and the C18 unsaturated fatty acids were concomitantly increased. The fae1 mutants were indistinguishable from wild type in seed physiology and plant growth. This study demonstrated that the CRISPR/Cas9 technology can be effectively applied to the polyploid crop camelina to rapidly obtain desired traits such as optimal fatty acid composition in its seed oil. Knocking out FAE1 also provides a means to increase the levels of oleic acid or $\alpha$-linolenic acid in camelina oils that are desirable for industrial or food/feed uses.
\end{abstract}

\section{Introduction}

Vegetable oils constitute one of the most important agricultural commodities. Vegetable oils are mainly consumed by humans, but considerable amounts are also used in industrial applications for manufacturing numerous products such as lubricants and biofuels (Gunstone, 1998). The final use of oils is dependent on their nutritional, physical and chemical properties. Camelina (Camelina sativa (L.) Crtz.), also known as false flax or gold-of-pleasure, is a member of the Brassicaceae family and was an established oilseed crop in parts of Eastern and Central Europe prior to World War II (Putnam et al., 1993). More recently, a strong interest has arisen in camelina as a potential biofuel crop in North America and other parts of the world due to its favorable attributes such as low-input production and tolerance to drought and cold temperatures (Pilgeram et al., 2007). Camelina seed has an oil content of $\sim 35 \%$ of dry mass for current varieties. Camelina oils are high $(\sim 50 \%)$ in polyunsaturated fatty acids (PUFA), especially $\alpha$-linolenic (18:3), an omega-3 fatty acid, and are thus also attracting attention for use in the production of health-promoting foods and feeds. However, the oil profiles of available camelina varieties (Table 1) are less than ideal for either food or non-food uses. For example, for use as biofuel and other products such as lubricants, it would be beneficial to increase the oxidative stability by reducing the content of highly unsaturated fatty acids (Jaworski and Cahoon, 2003). In contrast, these PUFAs are highly desirable from a nutritional standpoint. Currently, the main source of omega-3 vegetable oils is from flax. Camelina provides an attractive alternative to this type of oil in the food and feed markets (Pilgeram et al., 2007). Camelina seeds contain significant amounts of C20-24 very long-chain fatty acids (VLCFAs), primarily eicosenoic acid (20:1) and lesser amounts of erucic acid (22:1). These VLCFAs need to be reduced for food/feed uses.

Camelina is an allohexaploid species, which could potentially complicate the process of breeding for desired traits due to gene redundancy and their complementary effects (Kagale et al., 2014; Hutcheon et al., 2010; Kang et al., 2011). Plant biotechnology, especially the recent advent of the highly efficient and facile CRISPR/Cas9 (clustered regularly interspaced short palindromic repeats/CRISPR-associated protein 9) system for precision gene modifications, provides an effective tool (Lowder et al., 2015). Camelina can be easily transformed by agrobacterium using a floral vacuum infiltration method, with 
Table 1

Fatty acid composition of a low-VLCFA camelina mutant compared with Suneson.

\begin{tabular}{lccccccccccc}
\hline & $16: 0$ & $18: 0$ & $18: 1$ & $18: 2$ & $18: 3$ & $20: 0$ & $20: 1$ & $20: 2$ & $20: 3$ & $22: 0$ & $22: 1$ \\
\hline Suneson & 5.8 & 1.8 & 17.3 & 20.7 & 33.0 & 1.4 & 14.4 & 1.4 & 1.0 & 0.4 & 2.8 \\
LE1914 & 6.7 & 3.0 & 22.4 & 24.1 & 35.1 & 0.8 & 6.1 & 0.7 & 0.5 & 0.3 & 0.4
\end{tabular}

Data show averages of seeds harvested from 6 plants.

transgenic seeds obtained in a relatively short period of time after infiltration (4-6 weeks) (Lu and Kang, 2008). This has made camelina a useful crop platform for biotechnology and translational research (Collins-Silva et al., 2011; Hines and Travis, 2016). Recent attempts of CRISPR/Cas9-induced gene mutagenesis in camelina have been successful (Jiang et al., 2017; Aznar-Moreno and Durrett, 2017; Morineau et al., 2017). Understanding genetic and biochemical mechanisms of oil biosynthesis is necessary to effectively modify plant oils for a wide range of applications (Napier and Graham, 2010).

In oilseeds, fatty acids synthesized from acetyl-CoA in the plastids are exported into the cytosol for triacylglycerol (TAG) assembly in the endoplasmic reticulum (ER) (Browse and Somerville, 1991). The newly made fatty acids, mainly oleic acid (18:1) and a small amount of palmitic acid (16:0) and stearic acid (18:0), are first incorporated into phosphatidylcholine (PC) by the de novo pathway (Kennedy, 1961). They may also be incorporated into PC by an acyl exchange mechanism known as "acyl editing" mediated primarily by the lysophospholipid acyl-CoA: acyltransferases (Bates et al., 2009, 2012). The oleic acid is further desaturated to form the polyunsaturated linoleic (18:2) and $\alpha$ linolenic (18:3) acids by the fatty acid desaturases FAD2 and FAD3 in the endoplasmic reticulum (ER) using the phosphatidylcholine (PC) substrate, or elongated into $20: 1$ and $22: 1$ by the fatty acid elongase FAE1 (Li-Beisson et al., 2013). Several other biosynthetic enzymes such as phosphatidylcholine diacylglycerol cholinephosphotransferase (PDCT), lysophosphatidylcholine acyltransferases (LPCAT), and diacylglycerol acyltransferases (DGAT) may also affect the fatty acid composition of TAG by regulating the acyl flux through its precursors including PC and diacylglycerols (Bates et al., 2012; Lu et al., 2009, 2011; Marmon et al., 2017). In this study, we demonstrated that mutations at the FAE1 genes in camelina significantly changed fatty acid composition by decreasing the VLCFA content. Knocking out all alleles of the FAE1 genes by the CRISPR-Cas9 technology almost eliminated the accumulation of these fatty acids in camelina seeds.

\section{Methods}

\subsection{Mutant isolation and characterization}

Camelina (Camelina sativa) cv. Suneson was used in this study. Camelina seeds were treated with ethyl methanesulfonate (EMS) to induce mutations as described previously (Büchsenschütz-Nothdurft et al., 1998). The M2 generation of the treatments was grown in the field at the Arthur Post Agronomy Farm at Bozeman, MT. Seeds harvested from individual M2 plants were analyzed for fatty acid composition in a high-throughput fashion as described (Lu et al., 2006). Mutant lines showing altered fatty acid composition were collected and grown for another generation. Lines of homogeneity for the low 20:1 content were determined by analyzing at least 10 plants. These lines were chosen for further investigation and sequencing of the FAE1 alleles. Genomic DNA was isolated from young camelina leaves using the method as described (Lukowitz et al., 2000).

\subsection{CRISPR/Cas9 vector construction}

The single guide RNA (sgRNA) targeting all three alleles of Camelina sativa Fatty Acid Elongase (CsFAE1-A, Csa11g007400; CsFAE1-B, Csa10g007610; and CsFAE1-C, Csa12g009060) (Hutcheon et al., 2010) was designed by using gRNA prediction algorithms of Harvard CHOPCHOP website (http://chopchop.cbu.uib.no) (Labun et al., 2016). The gRNA was selected according to the following criteria: (1) The proximity to the $5^{\prime}$ end, (2) the coverage of all three CsFAE1 genes, (3) the higher efficiency score, (4) the absence of predicted possible off-targets, (5) minimum self-complementary regions, (6) higher GC content, preferably, between 40 and 70\% (Wang et al., 2014; Tsai et al., 2015). The best gRNA that obeys the criteria above was then tested with Cas-OFFinder web-based software (http://www.rgenome.net/cas-offinder) for off-target possibility (Bae et al., 2014). CsFAE1 sgRNA was synthesized by Eurofins MWG Operon USA (Louisville, $\mathrm{KY}$ ) as two oligonucleotides: gRNA-F, 5'-ATTGTTGGAGATGGGAATAGAAG-3', and gRNA-R, 5'AAACCTTCTATTCCCATCTC CAA-3' (sgRNA is underlined). Two oligos were mixed and incubated in a thermocycler at $95^{\circ} \mathrm{C}$ for $5 \mathrm{~min}$ and then cooled down to $4{ }^{\circ} \mathrm{C}$ for $5 \mathrm{~min}$. The FAE1 gRNA was then cloned into the binary transformation vector pHEE401E (Xing et al., 2014; Wang et al., 2015) (obtained from Addgene; www.addgene.org/71827) by the Golden Gate cloning method (Engler et al., 2008). Finally, the sgRNACas 9 cassette was digested with HindIII and EcoRI, then the entire sgRNA-Cas9 cassette was ligated into the pBinGlyRed2 vector ( $\mathrm{Lu}$ and Kang, 2008). In the final construct, sgRNA is driven by Arabidopsis U626 promoter (Li et al., 2007), then followed by gRNA scaffold and U626 terminator. Maize codon optimized Cas 9 (zCas9) gene is under the control of the egg-cell specific enhancer (EC1.2en) and promoter (EC1.1pro), and transcription ends with Pisum sativum rbcS-9E terminator (Wang et al., 2015) (Fig. 1).

\subsection{Camelina transformation}

The above construct was transformed into Agrobacterium strain GV3101 (pMP90) by electroporation with $0.2 \mathrm{~cm}$ cuvettes (Bio-Rad, Hercules, CA, USA) at a field strength of $2.5 \mathrm{kv} / \mathrm{cm}$, restorors of $600 \Omega$ and a capacitance of $25 \mu \mathrm{F}$. Selection of transformed bacteria was carried out on LB plates containing $50 \mu \mathrm{g} / \mathrm{ml}$ kanamycin and $40 \mu \mathrm{g} / \mathrm{ml}$ gentamycin. Camelina plants were grown in 6 " pots (5 plants/per pot) filled with 1:1 mix of MSU soil (equal parts by volume of loam soil: washed concrete sand: Canadian sphagnum peat moss with AquaGro, $2000 \mathrm{G}$ wetting agent blended in at $1 \mathrm{lb} /$ cubic yard of soil, aerated steam pasteurized at $70{ }^{\circ} \mathrm{C}$ for $1 \mathrm{~h}$ ) and Sunshine Mix \#1 (Bellevue, WA, USA). Greenhouse conditions were $22 / 18 \pm 1{ }^{\circ} \mathrm{C}$ for day/night temperatures, a relative humidity of $30 \%$, and a $16 \mathrm{~h}$ photoperiod of natural lighting supplemented when necessary by season. A well-established camelina transformation protocol developed at our laboratory was followed to generate transgenic plants ( $\mathrm{Lu}$ and Kang, 2008). Transgenic DsRed-expressing seeds were screened by illuminating mature seeds with green LED lights and viewing through a red filter. Transgenic plants were confirmed by PCR using the U6-specific primers (Suppl. Table 1) and genomic DNA extracted from leaves of 15-day old plants by the CTAB method (Lukowitz et al., 2000).

\subsection{Seed and fatty acid analyses}

For fatty acid analysis, camelina seeds were crushed and lipids were transmethylated according to procedures described previously (Lukowitz et al., 2000). The resulting fatty acid methyl esters (FAMEs) were analyzed in a gas chromatograph (GC) (Shimadzu Scientific Instruments, Columbia, MD) fitted with a $15 \mathrm{~m} \times 0.25 \mathrm{~mm}$ column (DB23; Agilent, Lexington, MA) and a flame ionization detector (FID). The GC was programmed for an initial temperature of $180{ }^{\circ} \mathrm{C}$ for $1 \mathrm{~min}$ followed by an increase of $10{ }^{\circ} \mathrm{C} / \mathrm{min}$ to $230^{\circ} \mathrm{C}$ and maintained for a further $1 \mathrm{~min}$.

Seed weight was determined by weighing 100 seeds. Seed oil content was determined by a bench-top nuclear magnetic resonance (NMR) seed analyzer (MQC23, Oxford Instruments, Concord, MA, USA) and by GC analysis using heptadecanoic acid (17:0) as internal standard (10 $\mathrm{mg} / \mathrm{ml}$ ), which was added to test tubes prior to FAMEs 
A

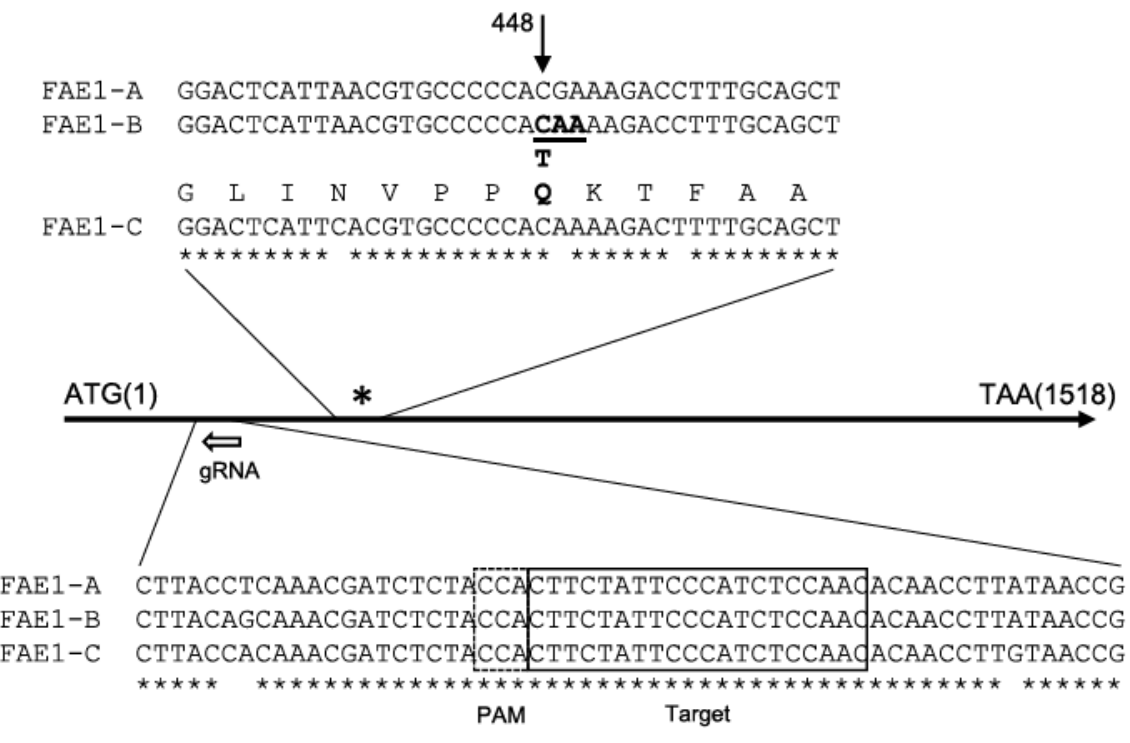

B

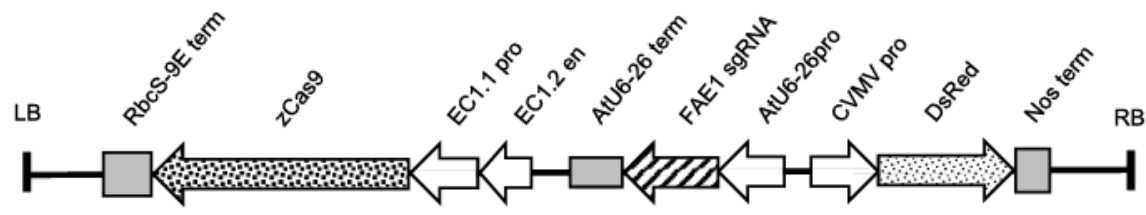

Fig. 1. Mutagenesis in the FAE1 alleles in Camelina sativa. (A) An EMS-induced mutation is shown on top at nucleotide no. 448 from the start codon ATG in the FAE1-B gene. Single-letter amino acids are encoded by FAE1-B. The sgRNA sequence targeting all three FAE1 genes (on the reverse strands) is shown at the bottom. Asterisks indicate identical nucleotides. PAM: protospacer adjacent motif. (B) T-DNA region (between the left/right borders) of the CRISPR/cas9 construct. The FAE1 sgRNA gene is bracketed by the Arabidopsis thaliana U6-26 small nuclear RNA promoter (U6-26 pro) and terminator (U6-26 term). zCas9 (Zea mays codon optimized) is driven by an egg cell-specific 1.2 enhancer (EC1.2 en) and an egg cell-specific 1.1 promoter (EC1.1 pro), and the translation stopped by a Pea sativum Rubisco small subunit 9E terminator (RbcS-9E term). The selection marker DsRed is driven by the cassava vein mosaic virus promoter (CVMV pro). derivatization. All seeds used for comparison of oil content and seed traits were harvested from plants grown at the same time in the greenhouse.

\section{Results and discussion}

\subsection{A low-VLCFA camelina line contains a mutation at the FAE1 locus}

In our previous experiments to isolate high-oleic camelina mutants, we screened an M2 population derived from ethyl methanesulfonate (EMS)-treated seeds (cultivar Suneson) and obtained several lines that have altered seed fatty acid profiles (Kang et al., 2011). One mutant line, which we designated as LE1914, showed a moderate increase in oleic acid (18:1). More significantly, the mutant seed contained reduced levels of very long-chain fatty acids comprising primarily of eicosenoic acid (20:1) and erucic acid (22:1). Compared to Suneson, which contained $17.2 \%$ of these fatty acids, the level decreased to only $6.5 \%$ in LE1914 (Table 1). The accumulation of eicosanoic acid (20:0) and other minor VLCFAs, was also significantly reduced in the mutant.

In oilseeds, VLCFA synthesis is catalyzed by the Fatty Acid Elongase1 (FAE1) acting on 18:1-CoA (Coenzyme A) (Millar and Kunst, 1997). Camelina sativa is a close relative to Arabidopsis thaliana (Beilstein et al., 2006; Al-Shehbaz et al., 2006). Like Camelina, Arabidopsis seed contains high amounts of VLCFAs, predominantly 20:1 (about $21 \% \mathrm{w} / \mathrm{w}$ of total fatty acids). The Arabidopsis mutant fae1 contains negligible amount $(<1 \%)$ of $20: 1$ in seed oils (Kunst et al., 1992). It has been shown previously that camelina contains three FAE1 genes and they share very high sequence similarity between these two species (Hutcheon et al., 2010). We sequenced the homology CsFAE1 genes, FAE1-A (Csa11g007400), FAE1-B (Csa10g007610) and FAE1-C (Csa12g009060), in camelina wildtype (Suneson) and the LE1914 mutant. The FAE1-B locus in the LE1914 had a nucleotide change at \#448 (C to T), which would convert the codon CAA for amino acid
Glutamine (Gln) into a stop codon TAA (Fig. 1). This result suggested that a single FAE1 gene mutation caused over $60 \%$ reduction in the 20:1 + 22:1 content compared to the wildtype. There was no mutation detected at the other two FAE1 genes, which are presumably responsible for the remaining VLCFAs in the mutant seed.

\subsection{Cas 9 transgenic plants targeting FAE1 genes caused decreased accumulation of VLCFAs}

Characterization of the LE1914 mutant suggests that three FAE1 genes may function in an additive fashion in camelina seeds. To better understand FAE1 enzymes in the polyploidy camelina seeds, we attempted to create FAE1 mutants by the CRISPR/Cas9 technology targeting all three alleles of the CsFAE1 genes.

Sequences of the $1518 \mathrm{bp}-$-long FAE1 genes (CsFAE1-A, -B, -C) are highly similar to one other (more than $96 \%$ identical) and to their orthologs from Arabidopsis (Hutcheon et al., 2010). To ensure that gene mutations would cause the loss of enzyme activities, we designed the single guide RNA (sgRNA) sequence near the 5 '-end of the FAE1 coding region (Fig. 1A). The sgRNA sequence was selected based on the camelina genome sequence to avoid potential off-target sites. Nearby single nucleotide polymorphisms (SNPs) between the three genes were present. These SNPs along with sequence variations within the promoter regions (Suppl. Fig. S1) would help determine whether all genes were efficiently targeted and mutated by the Cas $9 /$ gRNA complex.

The sgRNA sequence was synthesized as two complementary oligonucleotides and inserted into $\mathrm{pHEE} 401 \mathrm{E}$ transformation vector. The sgRNA-Cas9 cassette was sub-cloned into the pBinGlyRed2 vector to use the DsRed as the selection marker. This construct was transformed into camelina plant (Suneson) using the Agrobacterium-mediated vacuum infiltration method. We obtained 14 transgenic seeds showing strong DsRed expression, which were successfully germinated in soil for further analysis. 


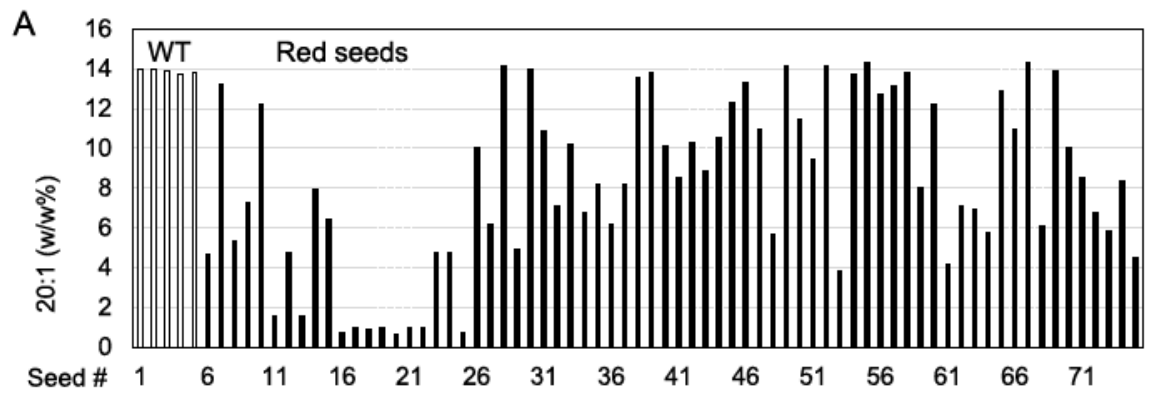

Fig. 2. Eicosenoic acid (20:1) content in single seeds of wild type (WT) and transgenic camelina. Seeds no. 1-5 (white bars) show wild type. Red (A) and brown (B) seeds harvested from $14 \mathrm{~T} 1$ transgenic lines are shown in black bars.

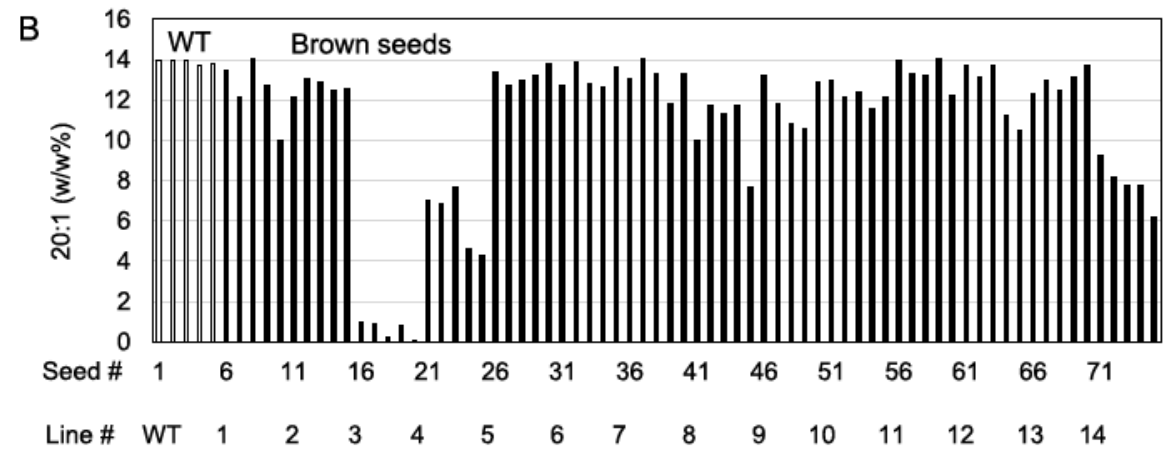

Seeds were harvested from all 14 independent T1 lines that showed DsRed segregation indicating single-transgene inserts (Suppl. Table 2). To evaluate the Cas9-induced mutations on FAE1 genes, five individual T2 seeds showing DsRed expression from each transgenic line were analyzed for their fatty acid composition by gas chromatography (Lu et al., 2006), and compared with non-transgenic seeds of Suneson that had been grown together with the transgenic plants in the greenhouse. We focused on the content of 20:1 since it's the most abundant VLCFA synthesized by the FAE1 enzyme in camelina. The wild type seeds accumulated about $14 \% 20: 1$ of total fatty acids (Seeds no. 1-5, Fig. 2). Of the 70 red seeds analyzed, 19 appeared to be wild type that had $12-14 \%$ of $20: 1$. The other 51 low-20:1 putative mutant seeds were from every transgenic line, showing a wide range of variation (ranging from 0.6 to $11.4 \% \mathrm{w} / \mathrm{w}$ ) (Fig. 2A). This result suggested that FAE1 genes have possibly been mutated by CRISPR/Cas9 in transgenic plants, and that FAE1 genes are responsible for most of 20:1 accumulation in camelina seed. The transgenic seeds fell into three major groups according to the level of $20: 1$ (around $5-11 \%, 2-5 \%$, and $<2 \%$ ). These might reflect the number (1,2 and 3) of FAE1 genes that were disrupted in their genomes.

Germline mutation was expected since the Cas 9 gene was driven by an egg cell-specific promoter. We speculated that FAE1 mutations might have occurred in brown (Cas9-negative) seeds, as well as in red seeds. We then analyzed five single brown seeds from each of the above 14 transgenic lines. As shown in Fig. 2B, low 20:1 seeds were detected from 7 lines, though at a lower frequency (20/70). Specifically, all seeds from line \#3 had less than $1 \%$ of $20: 1$, indicating that this line had already reached homozygosity of the fae 1 knockout mutation. Our results thus demonstrated that homozygous camelina mutants could be obtained in a single generation, as previously reported for Arabidopsis using the same egg cell-specific promoter-driven CRISPR/Cas 9 system (Wang et al, 2015).

\subsection{Mutations at the FAE1 alleles in the Cas9 transgenic camelina plants}

To assess the efficacy of Cas9-induced mutagenesis at the FAE1 gene sequences, genomic sequences near the sgRNA sites were examined in transgenic plants. DNA fragments flanking the three FAE1 genes present in the A, B and C subgenomes of the allohexaploid camelina were amplified by three pairs of gene-specific primers (Suppl. Fig. S1). PCR products from transgenic plants for all three genes were indistinguishable in size from those amplified from Suneson, suggesting no large insertion/deletion occurred at FAE1 alleles by the Cas9/sgRNA expression. Sequencing of the three PCR fragments amplified from the wildtype plants resulted in sequences identical to the previously published (Kagale et al., 2014; Hutcheon et al., 2010), indicating that the primers were able to specifically amplify the three pairs of the FAE1 alleles in the camelina subgenomes.

For each FAE1 gene, 7 of the $14 \mathrm{~T} 1$ plants did not contain any nucleotide changes at the target sites. In the remaining 7 transgenic T1 plants, nucleotide deletions or substitutions were detected at the sgRNA target sites in at least one of the three FAE1 genes (Suppl. Fig. S2). We planted 10 red seeds from each T1 line to grow into T2 plants. Leaf samples of each plant were collected and stored in $-80{ }^{\circ} \mathrm{C}$ for later analysis by extracting genomic DNA and sequencing. Putative homozygous T2 lines, determined by all red seeds they produced, were obtained. We selected one homozygous plant from each of the T2 lines to examine the Cas9-induced mutations at the FAE1 genes. These plants were confirmed to have the Cas9 transgenes by PCR using the U6specific primers (Suppl. Table 1). Sequencing results indicated that nucleotide deletions at the predicted locations near PAM sites occurred in 13 of the 14 lines for each of the FAE1 genes. Nucleotide substitutions were also detected in 10 plants for each gene (Suppl. Fig. S3).

The high frequency of mutagenesis in the T2 plants compared to T1 plants was consistent with previous studies (Jiang et al., 2017; Morineau et al., 2017), suggesting continued Cas9/sgRNA actions as transgenic plants were advanced to the next generations. Combining with fatty acid analysis (Fig. 2), our sequencing results also indicated that homozygous Cas9-transgenic camelina plants carrying mutations at all three FAE1 alleles have already been obtained in the T2 generation. Although the mutations affected all FAE1 genes in $13 \mathrm{~T} 2$ lines, results from Fig. 2 suggested that not all mutations would cause FAE1 inactivation. Some of the nucleotide deletions or substitutions (Suppl. Fig. S3) that led to deletion or substitution of 1-2 amino acids in the translated FAE1 proteins may not significantly affect their enzyme activities, therefore near or equal to wild type-level 20:1 contents were 
WT

Line 3-3-1

Line $3-3-3$

Line $3-3-4$

Line $3-3-14$
СCTCAAACGATCTCTACCACTTCTATTCCCATCTCCAACACAACCTTATAACCGTAATTT

$$
\begin{array}{llllll}
Y & H & F & Y & S
\end{array}
$$

CCTCAAACGATCTCTACCAC-----TTCCCATCTCCAACACAACCTTATAACCGTAATTT

CCTCAAACGATCTCTACCC--TTTATTCCCATCTCCAACACAACCTTATAACCGTAATTT

CCTCAAACGATCTCT-CC-CTTCTTTATTCCCATCTCCAACACAACCTTATAACCGTAATTT

$$
S \quad L \quad L \quad Y \quad S
$$

FAE1-B (Csa10g007610)

WT CAGCAAACGATCTCTACCACTTCTATTCCCATCTCCAACACAACCTTATAACCGTAATTT

Line 3-3-1

Line $3-3-3$

Line $3-3-4$ CAGCAAACGATCTCTACCACTT-TATTCCCATCTCCAACACAACCTTATAACCGTAATTT CAGCAAACGATCTCT------------CATCTCCAACACAACCTTATAACCGTAATTT CAGCAAACGATCTCTACCCCT--TATTCCCATCTCCAACACAACCTTATAACCGTAATTT

Line $3-3-14$
$-5$

$-2$

$-2,+2$

$-2$

FAE1-C (Csa12g009060)

WT

Line $3-3-1$

Line $3-3-3$

Line $3-3-4$

Line $3-3-14$
CCACAAACGATCTCTACCACTTCTATTCCCATCTCCAACACAACCTTGTAACCGTAATTT CCACAAACGATCTCTACCACT-----TCCCATCTCCAACACAACCTTGTAACCGTAATTT CCACAAACGATCTCTACCACTT-TATTCCCATCTCCAACACAACCTTGTAACCGTAATTT CCACAAACGATCTCTACCACTT-TATTCCCATCTCCAACACAACCTTGTAACCGTAATTT CCACAAACGATCTCTACCACT--TATTCCCATCTCCAACACAACCTTGTAACCGTAATTT
$-1$

$-14$

$-2$

$-13$

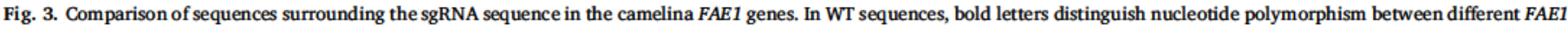

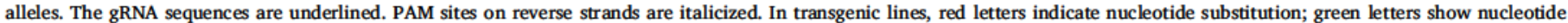

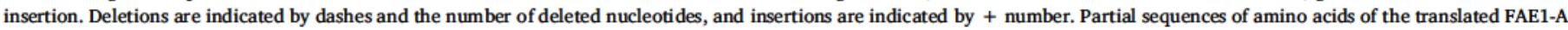
proteins are also shown under the nucleotide sequences.

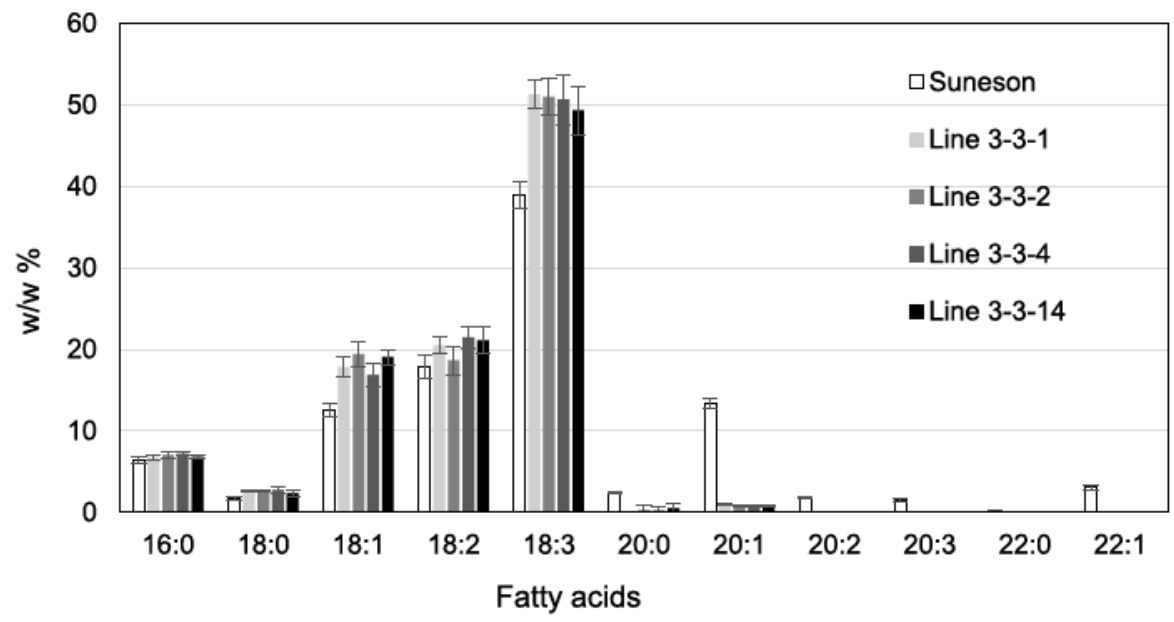

Fig. 4. Comparison of fatty acid composition between the best performing Cas 9 transgenic seeds and the non-transgenic wildtype camelina (Suneson). Data are means and standard errors from 5 single seeds harvested from 3 individual T3 plants grown in the greenhouse at the same time.

measured in those lines.

We reasoned that the seeds containing the lowest amounts of VLCFAs may have been caused by knockout mutations at all FAE1 alleles. To test this possibility, we selected a putative homozygous T2 line (\#3-3) that all 5 red seeds analyzed contained low amounts of 20:1. We excised small chips from the cotyledons of 20 red seeds for analyzing fatty acid composition. The seeds contained less than $1 \%$ of $20: 1$ were selected to germinate and gave rise to T3 plants (3-3-1, 3-3-3, 3-3-4 and 3-3-14) for sequence analysis. As expected, all three alleles of FAE1 were mutated at the sgRNA target sites, primarily by deletions near the PAM sites that would cause translational frameshift and resulted in FAE1 enzyme inactivation (Fig. 3). An exception was in the FAE1-A gene in line 3-3-4, where the deletion was compensated by the insertion of two nucleotides so that the gene might still be translated into an intact protein. However, the substitution of the amino acids Tyr-HisPhe in the wildtype FAE1-A with Ser-Leu-Leu might render the mutant enzyme inactive.

Due to limited number of sequences generated in this study, we did not attempt to assess the overall efficiency or the nature of the mutants (such as insertions, deletions, or nucleotide substitutions), which have been extensively evaluated in previous studies in camelina (Jiang et al., 2017; Morineau et al., 2017). However, our results clearly indicated that the Cas9/sgRNA effectively induced gene mutations at the FAE1 targets, and the desired fatty acid changes can be obtained from a small number (e.g. 14) of transgenic plants. Homozygous knockout mutants of multiple genes in the allohexaploid camelina can be obtained as early as the T2 generation by using an egg-cell specific promoter for Cas9/ sgRNA expression. 


\subsection{Improving fatty acid composition in camelina seeds using FAE1 mutagenesis}

Analyses of fatty acid composition of the Cas9-transgenic homozygous seeds indicated that knocking out all the FAE1 alleles significantly reduced the VLCFAs. In one of the best performing lines (\#33 ), these C20 fatty acids comprised of less than $1.5 \%$ of total fatty acids compared to over $22 \%$ in the wildtype seeds (Fig. 4). This result was similar to the Arabidopsis fae1 mutant (Kunst et al., 1992). The small amounts of VLCFAs in these mutants are possibly due to actions by other factors that are non-allelic to FAE1 (Kunst et al., 1992). The decrease of VLCFAs caused concomitant increases in other fatty acids (C16 and C18), especially the unsaturated fatty acids including oleic (18:1), linoleic (18:2) and $\alpha$-linolenic (18:3) acids. This is expected since the elongation and desaturation are the two major pathways for 18:1 modification. Knocking out FAE1 would block the elongation pathway and direct fatty acyl flux toward desaturation. Our results demonstrate that Cas9/sgRNA targeting the FAE1 genes can be effectively applied to make changes in camelina seed for favorable fatty acid compositions. For instance, one of the desirable traits in camelina seeds is its high concentration of $\alpha$-linolenic acid (18:3) for food and feed application. Eliminating VLCFAs increased 18:3 content from $39 \%$ in Suneson to $50 \%$ in the best Cas 9 transgenic seed grown in our greenhouse conditions (Fig. 4).

Shutting off the 18:1 elongation pathway by knocking out FAE1 may provide a tool to increase the oleic acid content for food and industrial applications. Reduction of 18:1 desaturation may be achieved by decreasing the FAD2 activity in camelina seed by RNA interference (RNAi) (Kang et al., 2011; Nguyen et al., 2013) or CRISPR/Cas9 technologies (Jiang et al., 2017; Morineau et al., 2017). Simultaneously knocking out all three FAD2 genes in camelina is however not feasible since unsaturated fatty acids are essential for plant growth. The Arabidopsis fad2 mutant failed to survive at low temperatures (Miquel et al., 1993). Camelina fad2 knockout mutants created by CRISPR/Cas 9 showed stunted growth (Morineau et al., 2017). It may be possible to target two of the FAD2 genes that are predominately expressed in seeds (Lowder et al., 2015) using gene-specific sgRNAs. By contrast, the camelina FAE1 knockout mutants did not show noticeable undesirable effects on seed physiology and plant growth. Seed germination and plant growth were comparable to Suneson when growing in normal greenhouse conditions. We analyzed transgenic T3 seeds for seed weight and oil content. These traits were similar to wildtype Suneson (Suppl. Fig. S4), and the mutant seeds were visually indistinguishable from the wild type. It therefore appears that FAE1 genes, which had been shown to be exclusively expressed in seed in Arabidopsis (Rossak et al., 2001) and camelina (Suppl. Table S3) (Kagale et al., 2016), are not essential for plant growth. We will further assess the effects of FAE1 mutations on plant performance in field conditions using homozygous fae1 knockout plants that are free of Cas9/sgRNA and DsRed transgenes.

\section{Conclusion}

The oilseed crop camelina has great potentials in food and non-food applications. It is necessary to improve fatty acid composition in its oils to meet different requirements. In this study, we investigated the usage of the Fatty Acid Elongase1 (FAE1) in camelina by traditional mutagenesis and new CRISPR/Cas9 approaches. A significant decrease in very long-chain fatty acids in a FAE1-B mutant suggested that the three FAE1 genes in camelina are responsible for synthesizing these VLCFAs and they may act in an additive fashion. By designing a sgRNA targeting all three FAE1 genes using the CRISPR/Cas9 technology, we obtained the knock-out mutants that accumulated very small amounts of VLCFAs without causing deleterious effects on seed traits and plant growth. Homozygous mutant plants were successfully obtained in a single generation by using an egg cell-specific expression of the Cas9/sgRNA transgenes. Our results demonstrated the high efficiency of the CRISPR/ Cas9 technology in a hexaploid oilseed crop to simultaneously edit the homologous genes. The FAE1 knock-out camelina seeds had significantly changed fatty acid profiles especially increased C18 unsaturated fatty acids, thus provide ways to breed high oleic acid or high $\alpha$-linolenic acid varieties for food or industrial applications.

\section{Contribution}

M.O., X.M., C.L designed experiments; M.O., X.M., J.K. conducted research; M.O and C.L. wrote the manuscript.

\section{Acknowledgements}

This project was partly funded by the US Department of Energy Office of Science (BER) grant no. DE-SC0016400, the National Science Foundation grant no. DBI 1339358, the Department of Agriculture grant no. 2015-67013-22815, and the Montana Agricultural Experimental Station.

\section{Appendix A. Supplementary data}

Supplementary data related to this article can be found at http://dx. doi.org/10.1016/j.plaphy.2017.11.021.

\section{References}

Al-Shehbaz, I.A., et al., 2006. Systematics and phylogeny of the Brassicaceae (Cruciferae): an overview. Plant Syst. Evol. 259, 89-120.

Aznar-Moreno, J.A., Durrett, T.P., 2017. Simultaneous targeting of multiple gene homeologs to alter seed oil production in Camelina sativa. Plant Cell Physiol. 58, 1260-1267.

Bae, S., et al., 2014. Cas-OFFinder: a fast and versatile algorithm that searches for potential off-target sites of Cas9 RNA-guided endonucleases. Bioinformatics 30, 1473-1475.

Bates, P.D., et al., 2009. Analysis of acyl fluxes through multiple pathways of triacylglycerol synthesis in developing soybean embryos. Plant Physiol. 150, 55-72.

Bates, P.D., et al., 2012. Acyl editing and headgroup exchange are the major mechanisms that direct polyunsaturated fatty acid flux into triacylglycerols. Plant Physiol. 160, 1530-1539.

Beilstein, M.A., et al., 2006. Brassicaceae phylogeny and trichome evolution. Amer. J. Bot. 93, 607-619.

Browse, J., Somerville, C., 1991. Glycerolipid synthesis - biochemistry and regulation. Annu. Rev. Plant Physiol. Plant Mol. Biol. 42, 467-506.

Büchsenschütz-Nothdurft, A., et al., 1998. Breeding for modified fatty acid composition via experimental mutagenesis in Camelina sativa (L.) Crtz. Ind. Crops Prod. 7, 291-295.

Collins-Silva, J.E., et al., 2011. Camelina: a designer biotech oilseed crop. Inform $610-613$.

Engler, C., et al., 2008. A one pot, one step, precision cloning method with high throughput capability. PLoS One 3, e3647.

Gunstone, F.D., 1998. Movements towards tailor-made fats. Prog. Lipid Res. 37, 277-305.

Hines, P.J., Travis, J., 2016. The new harvest. Science 353, 1218.

Hutcheon, C., et al., 2010. Polyploid genome of Camelina sativa revealed by isolation of fatty acid synthesis genes. BMC Plant Biol. 10, 233.

Jaworski, J., Cahoon, E.B., 2003. Industrial oils from transgenic plants. Curr. Opin. Plant Biol. 6, 178-184.

Jiang, W.Z., et al., 2017. Significant enhancement of fatty acid composition in seeds of the allohexaploid, Camelina sattiva, using CRISPR/Cas9 gene editing. Plant Biotechnol. J. $15,648-657$.

Kagale, S., et al., 2014. The emerging biofuel crop Camelina sativa retains a highly undifferentiated hexaploid genome structure. Nat. Commun. 5, 3706.

Kagale, S., et al., 2016. The developmental transcriptome atlas of the biofuel crop Camelina sativa. Plant J. 88, 879-894.

Kang, J., et al., 2011. Identification of three genes encoding microsomal oleate desaturases (FAD2) from the oilseed crop Camelina sativa. Plant Physiol. Biochem. 49, 223-229.

Kennedy, E.P., 1961. Biosynthesis of complex lipids. Fed. Proc. Amer. Soc. Exp. Biol. 20, 934-940.

Kunst, L., et al., 1992. Fatty acid elongation in developing seeds of Arabidopsis thaliana. Plant Physiol. Biochem. 30, 425-434.

Labun, K., et al., 2016. CHOPCHOP v2: a web tool for the next generation of CRISPR genome engineering. Nucleic Acids Res. 44, W272-W276.

Li, X., et al., 2007. Varied transcriptional efficiencies of multiple Arabidopsis U6 small nuclear RNA genes. J. Integr. Plant Biol. 49, 222-229.

Li-Beisson, Y., et al., 2013. Acyl-lipid Metabolism. The Arabidopsis Book/American Society of Plant Biologists, pp. 11. 
Lowder, L.G., et al., 2015. A CRISPR/Cas9 toolbox for multiplexed plant genome editing and transcriptional regulation. Plant Physiol. 169, 971-985.

Lu, C., Kang, J., 2008. Generation of transgenic plants of a potential oilseed crop Camelina sativa by Agrobacterium-mediated transformation. Plant Cell Rep. 27, 273-278.

Lu, C., et al., 2006. A high-throughput screen for genes from castor that boost hydroxy fatty acid accumulation in seed oils of transgenic Arabidopsis. Plant J. 45, 847-856.

Lu, C., et al., 2009. An enzyme regulating triacylglycerol composition is encoded by the ROD1 gene of Arabidopsis. Proc. Natl. Acad. Sci. U. S. A. 106, 18837-18842.

Lu, C., et al., 2011. New frontiers in oilseed biotechnology: meeting the global demand for vegetable oils for food, feed, biofuel, and industrial applications. Curr. Opin. Biotechnol. 22, 252-259.

Lukowitz, W., et al., 2000. Positional cloning in arabidopsis. Why it feels good to have a genome initiative working for you. Plant Physiol. 123, 795-805.

Marmon, S., et al., 2017. Two acyltransferases contribute differently to linolenic acid levels in seed oil. Plant Physiol. 173, 2081-2095.

Millar, A.A., Kunst, L., 1997. Very-long-chain fatty acid biosynthesis is controlled through the expression and specificity of the condensing enzyme. Plant J. 12, 121-131.

Miquel, M., et al., 1993. Arabidopsis requires polyunsaturated lipids for low-temperature survival. Proc. Natl. Acad. Sci. U. S. A. 90, 6208-6212

Morineau, C., et al., 2017. Selective gene dosage by CRISPR-Cas9 genome editing in hexaploid Camelina sativa. Plant Biotechnol. J. 15, 729-739.
Napier, J.A., Graham, I.A., 2010. Tailoring plant lipid composition: designer oilseeds come of age. Curr. Opin. Plant Biol. 13, 330-337.

Nguyen, H.T., et al., 2013. Camelina seed transcriptome: a tool for meal and oil improvement and translational research. Plant Biotechnol. J. 11, 759-769.

Pilgeram, A.L., et al., 2007. Camelina sativa, a Montana omega-3 and fuel crop. In: Janick, J., Whipkey, A. (Eds.), Issues in New Crops and New Uses. ASHS Press, Alexandria, VA, pp. 129-131.

Putnam, D.H., et al., 1993. Camelina: a promising low-input oilseed. In: Janick, J., Simon, J.E. (Eds.), New Crops. Wiley, New York, pp. 314-322.

Rossak, M., et al., 2001. Expression of the FAE1 gene and FAE1 promoter activity in developing seeds of Arabidopsis thaliana. Plant Mol. Biol. 46, 717-725.

Tsai, S.Q., et al., 2015. GUIDE-seq enables genome-wide profiling of off-target cleavage by CRISPR-Cas nucleases. Nat. Biotechnol. 33, 187-197.

Wang, T., et al., 2014. Genetic screens in human cells using the CRISPR-Cas9 system, Science $343,80-84$.

Wang, Z.P., et al., 2015. Egg cell-specific promoter-controlled CRISPR/Cas9 efficiently generates homozygous mutants for multiple target genes in Arabidopsis in a single generation. Genome Biol. 16, 144.

Xing, H.L., et al., 2014. A CRISPR/Cas9 toolkit for multiplex genome editing in plants. BMC Plant Biol. 14, 327. 